\title{
Discrimination Method of Ship and Corner Reflector Based on Micro-Doppler Feature
}

\author{
Zhiyu Tao a, Qiang Fu \\ School of Electronic Science and Engineering, National University of Defense Technology ATR Key \\ Laboratory, Changsha, Hunan, 410073, China \\ a594035410@qq.com
}

\begin{abstract}
Corner reflector is a common passive electronic countermeasure, which has the advantages of low cost, high effectiveness, etc. In this paper, the complex motion scattering point with translation, acceleration and vibration has been established in order to solve the problem of identifying ship and corner reflector, their micro Doppler features are analyzed, the different micro motion characteristics of ship an corner reflector are compared, then proposes a method to identify ship and corner reflector based on the difference of the characteristics of the micro Doppler, simulation data and real data demonstrates the effectiveness of this method.
\end{abstract}

Keywords: Ship; Corner reflector; Micro-Doppler.

\section{Introduction}

Corner reflector is a typical passive jamming in radar electronic countermeasure environment. The corner reflector is disposed at a predetermined position or towed by ship to form strong echo. Corner reflector has the advantages of low cost, high effectiveness. It is easy to produce and equip, and can disturb a variety of radar system. Therefore, it has been widely used in electronic defensive confrontation. According to the open literature, the research on the characteristics of corner reflector is relatively abundant, but the method of anti-corner reflector is few. In [1], a Krogager polarimetric decomposition algorithm was proposed for the identification of ship and corner reflector. In [2], a variety of polarization decomposition-based methods are discussed, and the difference of polarimetric characteristics between ship and corner reflector is analyzed. In Literature [3], a corner reflector identification method based on the micro Doppler feature is proposed and their micro-motion characteristics are analyzed. However, in order to obtain the polarization information of the target, it is needed to increase the complexity of the radar system. On the other hand, due to the dynamics of sea clutter and the variation of its spectrum, the anti-corner reflector method based on micro Doppler features is also affected. Therefore, how to discriminate corner reflector interference from real maritime targets more effectively has become a recognized problem.

The concept of micro motion and micro Doppler provides a new solution to this problem. The US Naval Research Laboratory V. C. Chen jog defined as a target or goal other than the part of the center of mass and rotational acceleration. Different forms of micro motion will produce different Doppler modulation on radar echo, which is called the micro Doppler effect, and the micro Doppler reflects the essential characteristics of the target motion. Micro motion feature is a unique performance form of target motion characteristics, micro Doppler features of different forms of exercise are obviously different, so can through the extraction of micro Doppler features to identify targets. The ship relative to the radar seeker has radial non-uniform linear motion, as translational motion and accelerated motion, at the same time because of the waves surging ship itself will have certain vibration, so the ship is composite motion translation, acceleration and vibration. Because the quality of ship volume is too large, the vibration is not obvious, so the fretting is not obvious; but corner reflector has light weight and small volume, the main form of exercise is vibration with the surging waves, so its micro characteristics is obvious. Therefore, it can be identify ship and corner reflector through the radar echo of the micro Doppler information. 


\section{Mathematical analysis of Doppler characteristics of ship}

In the classical study of micro motion, the single form of motion is considered for each scattering point, but most of the ground targets and sea surface targets are not suitable for this model. In the actual scene, many of the goals of the movement is often not a single form, such as ship targets relative to the radar seeker has radial non-uniform linear motion, as translational motion and accelerated motion, at the same time because of the waves surging ship itself will have certain vibration, so the ship is composite motion translation, acceleration and vibration.

The ship motion model is as follows:

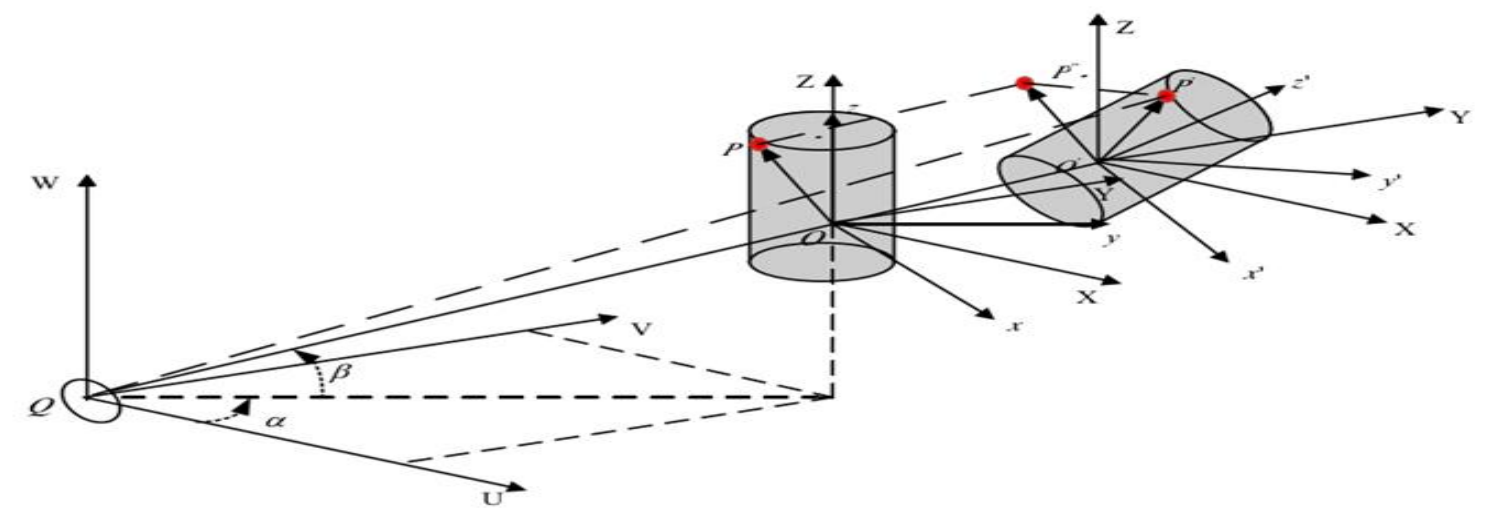

Fig.1 Ship motion model

In the $\mathrm{Q}$ for the origin of the radar coordinates $(\mathrm{U}, \mathrm{V}, \mathrm{W})$, it remained motionless; with $\mathrm{O}$ as the origin of the radar reference coordinates $(X, Y, Z)$; with $\mathrm{O}$ as the coordinate origin of target coordinates. The reference coordinate system is always kept in parallel with the radar coordinate system, the target coordinate system and the reference coordinate system origin coincide, and the coordinate origin of the reference coordinate system is $O^{\prime}$. The initial position vector of the reference coordinate system origin $\mathrm{O}$ in the radar coordinate system is $\mathbf{R}_{0}=\left(U_{0}, V_{0}, W_{0}\right)^{T}$, the initial azimuth and pitching angles are $\alpha, \beta$, and radar to the target radial unit vector is :

$$
\mathbf{n}=\mathbf{R}_{\mathbf{0}} /\left\|\mathbf{R}_{\mathbf{0}}\right\|=\left(\cos \alpha_{p} \cos \beta_{p}, \sin \alpha_{p} \cos \beta_{p}, \sin \beta_{p}\right)^{T}
$$

A target scattering point at the initial time of $\mathrm{t}=0$ when the position is $\mathrm{P}$, the corresponding position vector is $\mathbf{r}_{\mathbf{0}}=\left(x_{0}, y_{0}, z_{0}\right)^{T}$, the speed is $\mathbf{V}$, the acceleration is $\mathbf{a}$, the frequency of sine vibration is $f_{v}$, the amplitude is $D_{v}$, azimuth angle and pitching vibration direction angle are $\alpha_{p}, \beta_{p}$, and vibration direction of the unit vector is :

$$
\mathbf{n}_{\mathbf{v}}=\left(\cos \alpha_{p} \cos \beta_{p}, \sin \alpha_{p} \cos \beta_{p}, \sin \beta_{p}\right)^{T}
$$

In the time $P^{\prime}$ in the radar coordinate system, the corresponding position vector is:

$$
\mathbf{R}(t)=\mathbf{R}_{\mathbf{0}}+\mathbf{r}_{\mathbf{0}}+\mathbf{V} t+1 / 2 \mathbf{a} t^{2}+D_{v} \sin \left(2 \pi f_{v} t\right) \mathbf{n}_{\mathbf{v}}
$$

The distance of Radar to $\mathrm{P}$ 'point is:

$$
R(t)=\|\mathbf{R}(t)\|=\left\|\mathbf{R}_{\mathbf{0}}+\mathbf{r}_{\mathbf{0}}+\mathbf{V} t+1 / 2 \mathbf{a} t^{2}+D_{v} \sin \left(2 \pi f_{v} t\right) \mathbf{n}_{\mathbf{v}}\right\|
$$

Assuming the radar electromagnetic wave is sine signal, then the baseband signal of radar echo can be expressed as:

$$
s(t)=\rho(x, y, z) \exp \left\{j 2 \pi f \frac{2 R(t)}{c}\right\}
$$

Make derivation to the phase function $\Phi(R(t))$ can get echo Doppler frequency:

$$
\begin{aligned}
f_{d} & =\frac{1}{2 \pi} \frac{d \Phi(R(t))}{d t}=\frac{2 f}{c} \frac{d R(t)}{d t} \\
& =\frac{2 f}{c}\left[\mathbf{V}+\mathbf{a} t+2 \pi f_{v} D_{v} \cos \left(2 \pi f_{v} t\right) \mathbf{n}_{\mathrm{v}}\right]^{T} \mathbf{n}_{p^{\prime}} \\
& =\frac{2 f}{c} \mathbf{V}^{T} \mathbf{n}_{p^{\prime}}+\frac{2 f}{c} \mathbf{a}^{T} \mathbf{n}_{p^{\prime}} t+\frac{4 f}{c} \pi f_{v} D_{v} \cos \left(2 \pi f_{v} t\right) \mathbf{n}_{\mathrm{v}}{ }^{T} \mathbf{n}_{p^{\prime}}
\end{aligned}
$$


Make compensation to speed V can get the micro Doppler:

$$
f_{m-d}=\frac{2 f}{c} \mathbf{a}^{T} \mathbf{n} t+\frac{4 f}{c} \pi f_{v} D_{v} \cos \left(2 \pi f_{v} t\right) \mathbf{n}_{\mathbf{v}}{ }^{T} \mathbf{n}
$$

(7) shows that, when the target hsa acceleration and vibration with translational motion characteristics at the same time, $f_{m-d}$ would get linear modulation, frequency modulation rate is proportional to the acceleration at the same time, with the time to do the cyclical change, changes in the same period as the period of the oscillation frequency and amplitude change is proportional to the amplitude and vibration.

If the radar works in the $\mathrm{X}$ band, frequency $\mathrm{f}=35 \mathrm{GHz}, \mathbf{R}_{\mathbf{0}}=(1000 \mathrm{~m}, 3500 \mathrm{~m}, 5000 \mathrm{~m})^{T}$, the target is moving toward the radar with $\mathrm{a}=2 \mathrm{~m} / \mathrm{s}^{2}$, Because of the large size of the ship, the vibration mode is not obvious.

Figure 2 (a) is spectrum through fast Fourier transform of echo signal, Figure 2 (b): the time-frequency analysis of the echo signal by the rearrangement smoothed pseudo Wigner-Ville distribution.

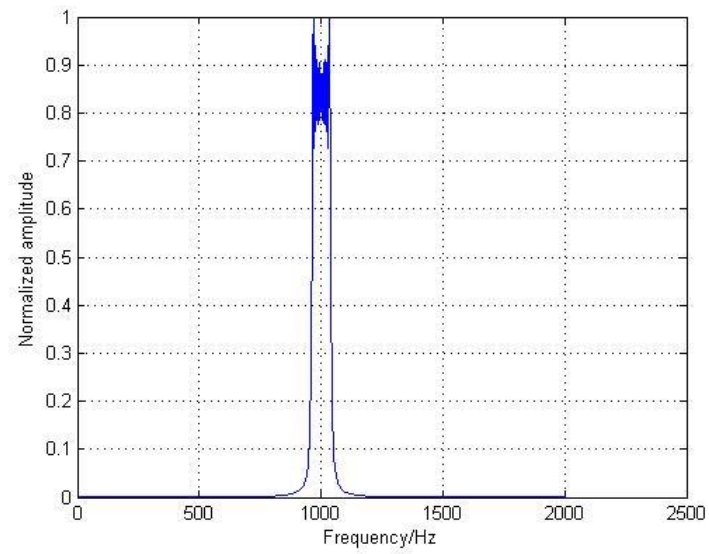

Fig.2 (a) Echo signal spectrum of ship

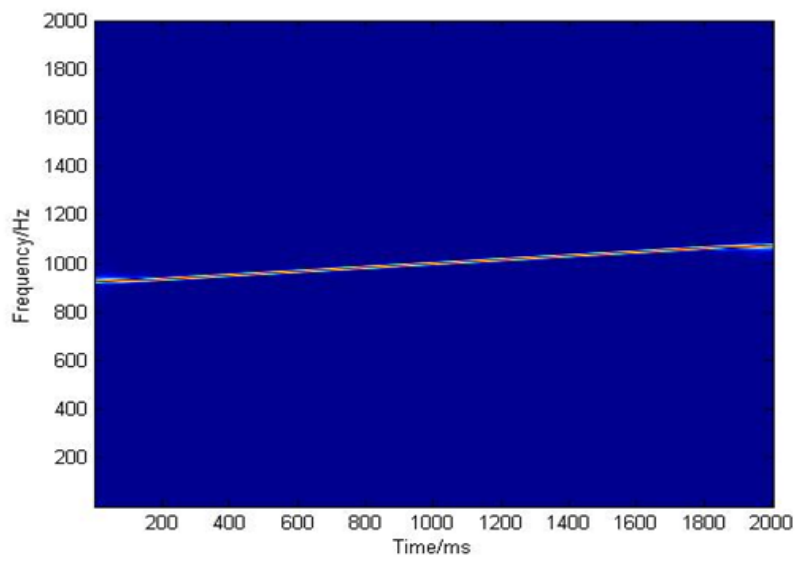

Fig.2 (b) Micro Doppler spectrum of ship

From Figure 2 (a), it can be seen that the spectrum of accelerated motion modulation has been broadened, but it can not be seen that the change of Doppler frequency with time. Figure 2 (b) not only can see the spectrum has been broadened, but also can see that the acceleration motion makes the micro Doppler frequency curve upward tilt, tilt and motion acceleration related, which is consistent with the results of theoretical derivation.

\section{Mathematical analysis of micro Doppler features of corner reflector}

Figure 3 is a schematic view of a corner reflector vibrations.

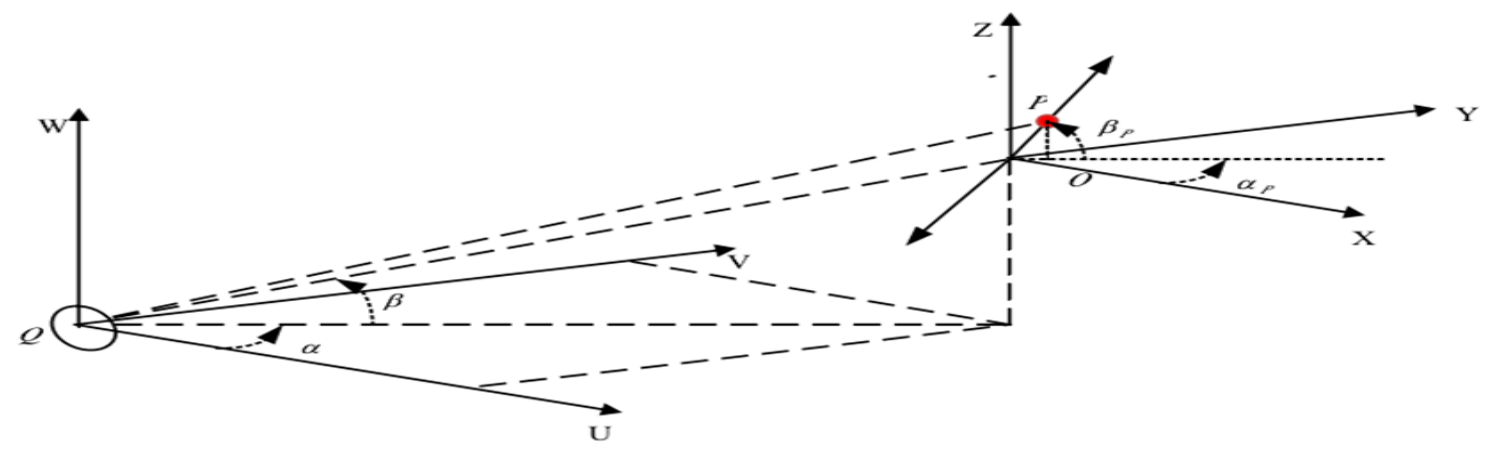

Fig.3 Ship motion model

In the $\mathrm{Q}$ for the origin of the radar coordinates $(\mathrm{U}, \mathrm{V}, \mathrm{W})$, it remained motionless; with $\mathrm{O}$ as the origin of the radar reference coordinates $(X, Y, Z)$; with $\mathrm{O}$ as the coordinate origin of target coordinates. The initial position vector of the reference coordinate system origin $\mathrm{O}$ in the radar 
coordinate system is $\mathbf{R}_{0}=\left(U_{0}, V_{0}, W_{0}\right)^{T}$, the initial azimuth and pitching angles are $\alpha, \beta$, and radar to the target radial unit vector is :

$$
\mathbf{n}=\mathbf{R}_{\mathbf{0}} /\left\|\mathbf{R}_{\mathbf{0}}\right\|=\left(\cos \alpha_{p} \cos \beta_{p}, \sin \alpha_{p} \cos \beta_{p}, \sin \beta_{p}\right)^{T}
$$

A target scattering point at the initial time of $\mathrm{t}=0$ when the position is $\mathrm{P}$, the corresponding position vector is $\boldsymbol{R}_{0}=\left(U_{0}, V_{0}, W_{0}\right)^{T}$, the frequency of sine vibration is $f_{v}$, the amplitude is $D_{v}$, azimuth angle and pitching vibration direction angle are $\alpha_{p}, \beta_{p}$, and vibration direction of the unit vector is :

$$
\mathbf{n}_{\mathbf{v}}=\left(\cos \alpha_{p} \cos \beta_{p}, \sin \alpha_{p} \cos \beta_{p}, \sin \beta_{p}\right)^{T}
$$

In the t time $P^{\prime}$ in the radar coordinate system, the corresponding position vector is

$$
\mathbf{R}(t)=\mathbf{R}_{\mathbf{0}}+D_{v} \sin \left(2 \pi f_{v} t\right) \mathbf{n}_{\mathbf{v}}
$$

The distance of Radar to $\mathrm{P}$ 'point is

$$
R(t)=\|\mathbf{R}(t)\|=\left\|\mathbf{R}_{\mathbf{0}}+D_{v} \sin \left(2 \pi f_{v} t\right) \mathbf{n}_{\mathbf{v}}\right\|
$$

Because of $R_{0}$ is much larger than $D_{v}$, Therefore, $\mathrm{R}(\mathrm{t})$ can be approximated

$$
R(t) \approx R_{0}+D_{v} \sin \left(2 \pi f_{v} t\right)\left(\cos \beta_{p} \cos \beta \cos \left(\alpha-\alpha_{p}\right)+\sin \beta \sin \beta_{p}\right)
$$

Assuming the radar electromagnetic wave is sine signal, then the baseband signal of radar echo can be expressed as:

$$
\begin{aligned}
s(t) & =\rho(x, y, z) \exp \left\{j 2 \pi f \frac{2 R(t)}{c}\right\} \\
& =\rho \exp \left\{R_{0}+D_{v} \sin \left(2 \pi f_{v} t\right)\left(\cos \beta_{p} \cos \beta \cos \left(\alpha-\alpha_{p}\right)+\sin \beta \sin \beta_{p}\right)\right\}
\end{aligned}
$$

Corner reflector scatters micro-Doppler features are:

$$
f_{m-d}=\frac{4 f}{c} \pi f_{v} D_{v} \cos \left(2 \pi f_{v} t\right)\left[\cos \beta_{p} \cos \beta \cos \left(\alpha-\alpha_{p}\right)+\sin \beta \sin \beta_{p}\right]
$$

The formula (14) shows that the micro Doppler characteristic of the corner reflector is a sine curve, whose period is the same as the vibration period, and its amplitude is related to the amplitude, frequency and vibration direction of the vibration.

If the radar works in the $\mathrm{X}$ band, frequency $\mathrm{f}=35 \mathrm{GHz}, \mathbf{R}_{\mathbf{0}}=(1000 \mathrm{~m}, 3500 \mathrm{~m}, 5000 \mathrm{~m})^{T}$, The scattering point $\mathrm{P}$ is doing sinusoidal motion with $D_{v}=0.1 \mathrm{~m}, f_{v}=1 \mathrm{~Hz}$, the azimuth and pitch angle of the vibration direction are respectively $\alpha_{p}=\pi / 9, \beta_{p}=\pi / 18$. Figure 4 (a) is spectrum through fast Fourier transform of echo signal, Figure 4(b): the time-frequency analysis of the echo signal by the rearrangement smoothed pseudo Wigner-Ville distribution.

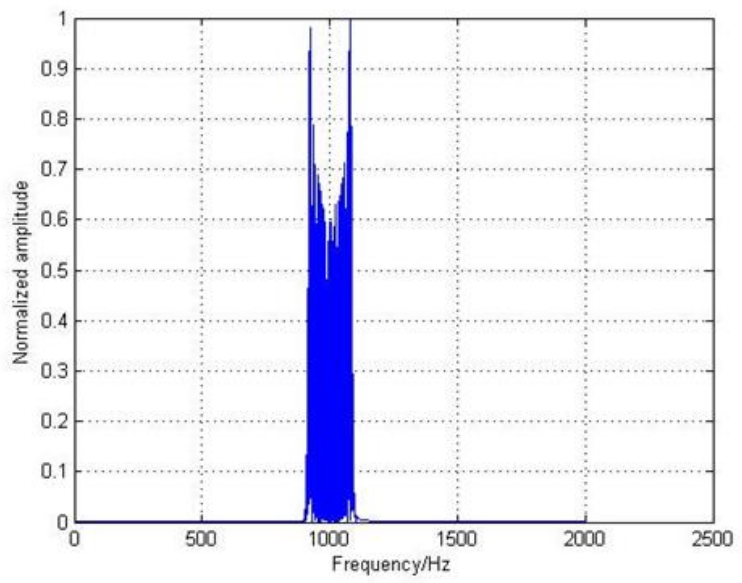

Fig.4 (a) Echo signal spectrum of corner reflector 


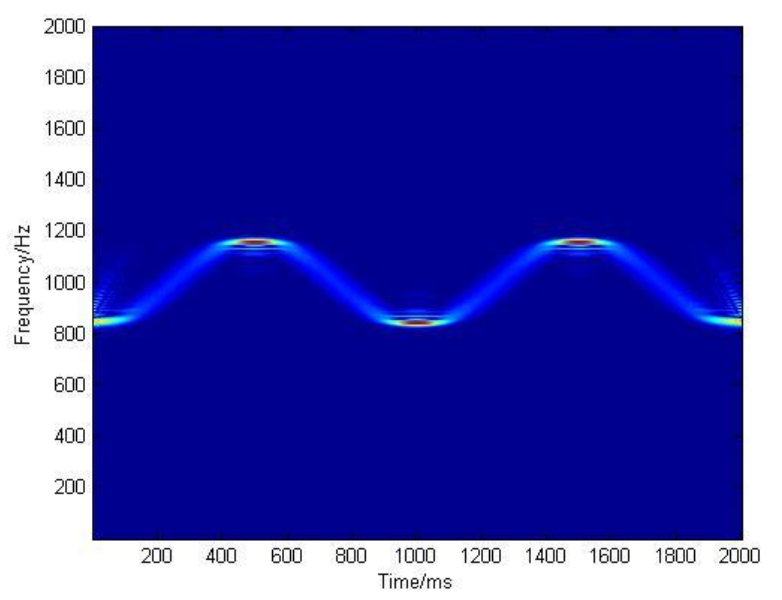

Fig.4 (b) Micro Doppler spectrum of corner reflector

From Figure 4(a), it can be seen that the spectrum of accelerated motion modulation has been broadened, but it cannot be seen that the change of Doppler frequency with time. Figure 4 (b) not only can see the spectrum has been broadened, at the same time, the time difference between the adjacent peaks of the micro Doppler curve in Figure 4 (b) can be seen that the period of the target vibration is about 1 second, which is consistent with the vibration frequency of $1 \mathrm{~Hz}$. The simulation results are consistent with the theoretical derivation.

\section{Validation through measured data}

The following uses a certain type of X-band single-polarized coherent seeker real data to evaluate algorithm. The seeker adopts the horizontal polarization mode, the range resolution is $15 \mathrm{~m}$, the pulse repetition rate is $1000 \mathrm{~Hz}$, and the pulse number is 2400 .

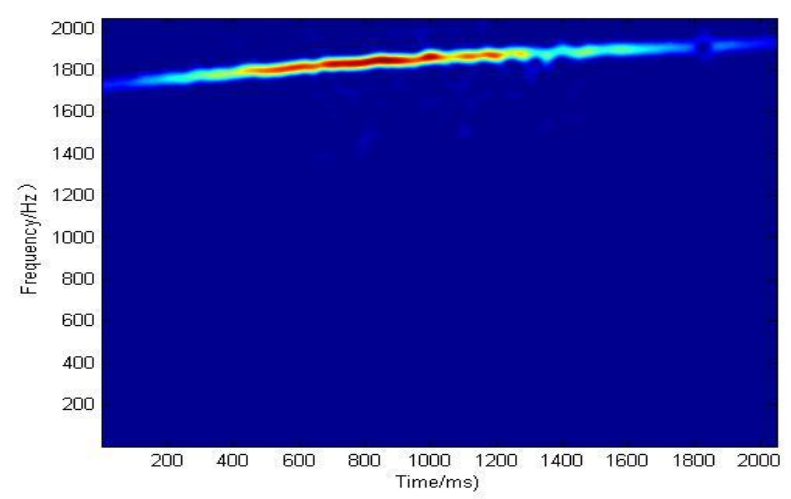

Fig.5 (a) Micro Doppler spectrum of ship measured da

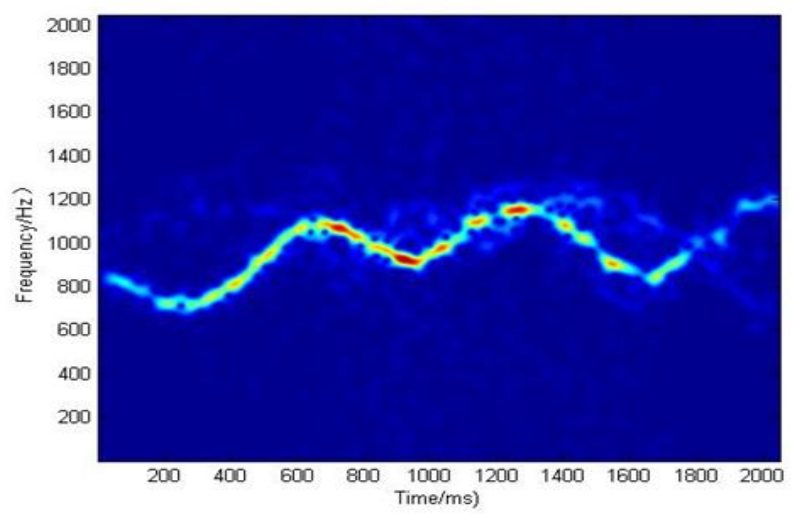

Fig.5 (b) Micro Doppler spectrum of corner reflector measured data 
By the results of the simulation and measured data can be seen, the micro-Doppler features of ship and corner reflector have obvious differences, it can be directly used to identify ship and corner reflector through micro Doppler echo signal characteristic differences.

\section{Summary}

Anti-jamming of corner reflector is a hot issue in the field of radar target recognition. In this paper, study the classification of the ship and the corner reflector in the narrow band low resolution radar system. Firstly, two types of target motion characteristics of theoretical has been analyzed, then establish micro model with translation, acceleration and vibration and analyze their micro Doppler features. Through the simulation experiment and the measured data, the ship and the corner reflector are obviously different from the micro Doppler feature, so it can be identify ship and corner reflector through the radar echo of the micro Doppler information .

\section{References}

[1] J. H. Tu, G. F. Tang, H. T. Xiao, "A study of anti-corner reflector based on ploarization decomposition," Radar Science and Technology, vol.7, no.2, pp.85-90, 2009.

[2] Z. Z. Zhu, G. F. Tang, Z. Cheng., "Discrimination method of ship and towed decoy based on polarization decomposition," Ship Board Electronic Counter Measure, vol.33, no.6, pp.15-21, 2010.

[3] M. J. Huang, H. Z. Zhao, Q. Fu, "A sea corner jamming identification method based on micro-Doppler feature,” Journal of Astronautics, vol.33, no.10, pp.1486-1491, 2012.

[4] F. Y. He, Z. Y. Xiao. Micro-motion modelling and analysis of extended ballistic targets based on inertial parameters [J]. Electronics Letters, 2013, 49(2): 129-130.

[5] S. Huixia, L. Zheng. Nutation feature extraction of ballistic missile warhead [J]. Electronics Letters, 2011, 47(13):770-772.

[6] H. Y. Yao, W. F. Sun, X. Y. Ma, et al. Precession feature extraction of warhead with empennages [J]. Electronics Letters, 2013, 49(9): 617-618. 